\title{
Asymptotic Methods for Thin High-Contrast Two-Dimensional PBG Materials
}

\author{
Wally Axmann, Peter Kuchment, and Leonid Kunyansky
}

\begin{abstract}
This paper surveys recent analytic and numerical results on asymptotic models of spectra of electromagnetic (EM) waves in two-dimensional (2-D) thin high-contrast photonic bandgap (PBG) materials. These models lead to discovery of interesting phenomena, including extremely narrow bands that can be used for spontaneous emission enhancement, gaps in the long wave regions, and asymptotic periodicity of the spectrum. The asymptotic results provide unexpectedly good qualitative (and sometimes quantitative) description of spectral behavior for materials of finite contrast. In some cases, simple ordinary differential models can be derived that yield a good approximation of the spectra. In such situations, one can obtain approximate analytic formulas for the dispersion relations.
\end{abstract}

Index Terms - Photonic bandgap (PBG), photonic crystal, spectrum.

\section{INTRODUCTION}

$\mathbf{P}$ HOTONIC bandgap materials (PBG; also called photonic crystals) have attracted a lot of attention since the idea was coined about a decade ago [39]. The reason is the wealth of expected (and partly achieved) applications. Probably the main feature of these materials is existence of a gap (stopband) in the frequency spectrum of propagating EM waves. There have been many successful experimental and numerical studies done in this area (see, for instance, books, bibliographies, and surveys ${ }^{1}$ [6], [11], [21], [23], [26], [27], [30], [35], [38], and references therein). It is unusual, however, that in spite of availability of a practically precise mathematical model (Maxwell equations), very little analytic study has been done. The main reason probably is that many problems that arise here are much more complex than in the case of the one particle Schrödinger operator, which is the main tool for similar studies in solid state physics [1]. One can mention, for instance, that a simple mechanism of creating spectral gaps by combining potential wells is absent in the case of the Maxwell operator, due to immobility of the lower edge of its spectrum. Some of these mathematical problems are addressed in the survey [27]. There is, however, at least one analytic approach that works. It has been acknowledged (see the surveys and books cited above) that thin structures of high dielectric contrast (like

Manuscript received March 18, 1999; revised August 17, 1999. This work was supported in part by the NSF through Grant DMS 9610444 and by the Department of Army, Army Research Office, through a DEPSCoR Grant.

W. Axmann and P. Kuchment are with the Mathematics and Statistics Department, Wichita State University, Wichita, KS 67260-0033 USA.

L. Kunyansky is with the Department of Applied Mathematics, California Institute of Technology, Pasadena, CA 91125 USA.

Publisher Item Identifier S 0733-8724(99)08808-8.

${ }^{1}$ Photonic and acoustic band-gap bibliography, Available http: http://www.ee.ucla.edu/labs/photon/biblio.html. air bubbles closely packed into an optically dense dielectric material) favor opening of spectral gaps. It is thus reasonable to undertake an asymptotic analysis of the problem, considering the width of the dielectric as a small parameter and the electric permittivity of the dielectric as a large one (certainly, interplay between these two parameters is also important). The hope is that if one can derive such an asymptotic model, it can give a lot of information. For instance, since the asymptotic model will probably be much simpler than the original one, one can expect some analytic rather than purely numerical results. One might be able to get better understanding of the qualitative (and maybe even quantitative) spectral behavior. There is also a hope to use the asymptotic spectra and eigenmodes as seeds for numerical methods for the full problem, for instance, in the finite element method as in [4] and [7]. The purpose of this paper is to survey some recent developments in this direction. We will confine ourselves to the practically important case of two-dimensional (2-D) photonic crystals and EM waves propagating along the periodicity plane. In this case, there are two possible polarizations (see [25], [26]). One is where the electric field $E$ is perpendicular to the plane of periodicity ( $E$-fields). Here, one deals with the scalar eigenvalue problem in $2-\mathrm{D}$

$$
-\Delta E=\lambda \varepsilon(x) E
$$

where $\lambda=(\omega / c)^{2}, \omega$ is the frequency of the wave, $c$ is the speed of light, and $\varepsilon(x)$ is the (periodic) electric permittivity function in the plane. The $H$-fields correspondingly yield the 2-D spectral problem

$$
-\nabla \cdot \frac{1}{\varepsilon(x)} \nabla H=\lambda H
$$

We want to mention that some spectral features are better visible in terms of the parameter $\lambda$ (sometimes after an appropriate rescaling), rather than in terms of the frequency $\omega$. That is why many our graphs are presented for $\lambda$ rather than $\omega$.

Probably the first asymptotic results for these problems were obtained in [12]-[15]. We briefly formulate some of them in Sections II and VI. In the case of $E$-fields one of the asymptotic regimes leads to the spectral problem for a first order pseudo-differential operator on a graph. Analysis of this asymptotic model of the $E$-fields is discussed in Section III. In particular, one observes interesting features like practical discreteness of the spectrum of disconnected structures, asymptotic periodicity of the spectrum, and existence of some extremely flat bands for some connected dielectric 


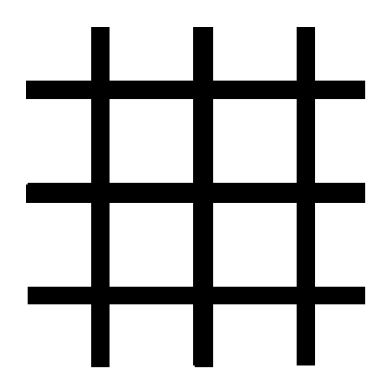

Fig. 1. 2-D square PBG structure of period 1. The dark strips of the width $\delta$ represent dielectric with $\varepsilon>1$. The white areas are filled with air $(\varepsilon=1)$.

structures. One also notices that the gaps can be opened for the waves much longer than the period of the structure. Analytic results explaining and predicting these effects are presented. An amazing possibility opens when one takes a second step of approximation (Section IV). Namely, the still complex asymptotic problem on a graph introduced in Section II can in some cases be very well approximated by a simple ordinary differential model, which resembles the "quantum mechanics on graphs" used in particular to describe quantum wires, thin superconducting structures, and free-electron models of organic molecules (see, for instance, [2], [3], [8]-[10], [19], [20], [31]-[34]). An example of such simplification is provided, where one can end up with simple explicit formulas for approximation of the band functions. Section V briefly mentions correspondence between the asymptotic and direct numerical results. One discovers that the asymptotic results can provide an unexpectedly good qualitative and even quantitative idea of the spectral effects. Section VI describes the asymptotic results for the $H$-fields. The last two sections contain the main conclusions and acknowledgment of the support and information the authors received from funding agencies and individuals.

\section{AN ASYMPTOTIC MODEL FOR THE E-FIELDS IN 2-D}

In this section, we consider waves polarized in such a way that the electric field $E$ is perpendicular to the plane of periodicity ( $E$-fields). Then the Maxwell system reduces to the scalar spectral problem

$$
-\Delta u=\lambda \varepsilon(x) u
$$

where $\lambda=(\omega / c)^{2}$. Most of the results will be presented in terms of the spectral parameter $\lambda$ rather than frequency $\omega$. The reason is that some effects are better visible in terms of $\lambda$. On the other hand, it is easy to translate everything into the frequency language.

The asymptotic study started with a thorough consideration of the simplest square geometry of a 2-D PBG medium (Fig. 1) undertaken in [12]-[14]. The medium is scaled to have period 1 in both $x$ and $y$ directions. The dark areas have thickness $\delta<1$ and are filled with a dielectric $(\varepsilon>1)$, while the light areas are filled with air $(\varepsilon=1)$. The square structure was chosen for its simplicity with the hope that studying it one can get better understanding of more complex geometries.

The asymptotic regime we are currently interested in is $\delta \rightarrow 0$ and $\varepsilon \delta \rightarrow \infty$. One can argue that these asymptotic

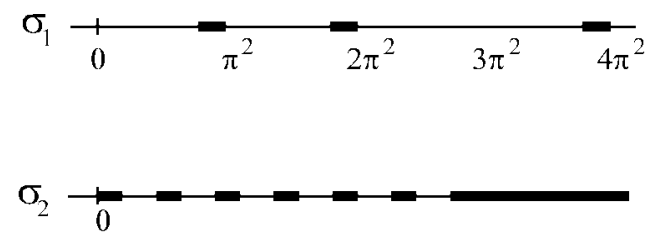

Fig. 2. The two subspectra for $E$-waves in the high-contrast square PBG structure.

assumptions are very far from what is currently technically feasible. Although this is true, there are amazing similarities between the nonasymptotic and asymptotic results, which will be discussed in Section V. Besides, later on we will relax the assumption $\varepsilon \delta \rightarrow \infty$ to $\varepsilon \delta \rightarrow$ const $>0$.

Denote by $S_{2}$ the spectrum of the Dirichlet Laplacian on the unit square

$$
S_{2}=\left\{\pi^{2}\left(n_{1}^{2}+n_{2}^{2}\right) \mid\left(n_{1}, n_{2}\right) \in Z^{2} \backslash\{(0,0)\}\right\} .
$$

Here, we adopt the standard notation $Z$ for the set of integers.

Theorem 1: (See more details in [13] and [14]) The spectrum $\sigma$ of the problem (1) for the square geometry splits into two parts: $\sigma=\sigma_{1} \cup \sigma_{2}$. If $\varepsilon \delta \rightarrow \infty$ and $\varepsilon \delta^{4 / 3} \longrightarrow 0$, then the following spectral asymptotics hold:

i) Any finite part $\sigma_{1} \cap[0, N]$ of the subspectrum $\sigma_{1}$ tends to the corresponding part $S_{2} \cap[0, N]$ of $S_{2}$. The (Hausdorff) distance between these two sets can be estimated as follows:

$$
d\left(S_{2} \cap[0, N], \sigma_{1} \cap[0, N]\right) \leq C_{N}(\varepsilon \delta)^{-1} .
$$

The corresponding eigenmodes are the "air modes."

ii) There exists a set of disjoint segments $\mathcal{D}=$ $\bigcup_{n>0}\left[\mathcal{D}_{n}^{-}, \mathcal{D}_{n}^{+}\right]$not depending on $\varepsilon$ and $\delta$ such that $\mathcal{D}_{0}^{-}=0$, $\mathcal{D}_{0}^{+}=4, \mathcal{D}_{n+1}^{-}>\mathcal{D}_{n}^{+}$, and $\mathcal{D}_{n}^{-} \sim 2 \pi n, \mathcal{D}_{n}^{-} \sim 2 \pi n+\pi$ when $n \rightarrow \infty$. Any finite part $\sigma_{2} \cap[0, N]$ of the subspectrum $\sigma_{2}$ behaves asymptotically as

$$
\left\{\bigcup_{n \geq 0}\left[(\varepsilon \delta)^{-1} \mathcal{D}_{n}^{-},(\varepsilon \delta)^{-1} \mathcal{D}_{n}^{+}\right]\right\} \cap[0, N]
$$

The corresponding eigenmodes are guided in the dielectric due to the total internal reflection.

This result shows that the subspectrum $\sigma_{1}$ of the air modes shrinks to the spectrum of the Dirichlet Laplacian on the unit square, therefore becoming almost discrete and opening large gaps at exactly described locations. A completely different behavior is observed in the second subspectrum $\sigma_{2}$ generated by the dielectric modes. Namely, it splits into narrow bands separated by narrow gaps, both of the asymptotic size $w=$ $(\varepsilon \delta)^{-1}$. Fig. 2 represents schematically these two spectra.

This theorem shows in particular that one can open an arbitrarily large number of spectral gaps for $E$-waves in highcontrast square structures. Another interesting observation is that one can open gaps for arbitrarily long waves without changing the periodicity scale of the structure. The dielectric $E$ modes, which are responsible for the subspectrum $\sigma_{2}$ present the main obstacle for the gaps opening, forcing the gaps to be small. 


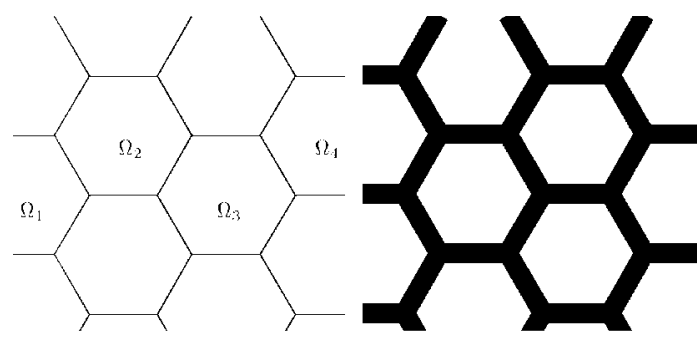

(a)

(b)

Fig. 3. (a) The graph $\Sigma$ and its faces $\Omega_{j}$. (b) The dielectric PBG material corresponding to the graph $\Sigma$. The dark areas of width $\delta$ represent dielectric with dielectric constant $\varepsilon>1$. The white areas are the air bubbles with $\varepsilon=1$.

The proof of the cited theorem relies on possibility of an approximation by a separable exactly solvable model. This is impossible for most geometries. So, one might wonder whether it is possible to carry over a similar analysis for nonsquare geometries. This question was answered in [15], where much more general periodic structures of the "fattened graph" type were studied. Consider a periodic graph $\Sigma$ on the plane that divides it into compact faces $\Omega_{j}$ [Fig. 3(a)]. Imagine that all its edges are fattened to the width $\delta$ [the dark areas in Fig. 3(b)] and filled with a dielectric with the dielectric constant $\varepsilon>1$. The rest of the plane (the white faces) is filled with air.

We will consider now the asymptotic behavior of the spectrum of the $E$ modes when $\delta \rightarrow 0$ and $\varepsilon \delta \rightarrow W^{-1}>0$. One can notice that while in the previous theorem we assumed that $W=0$, we now allow nonzero finite limits of $\varepsilon \delta$. This is a much more realistic assumption, since the values of $\varepsilon \delta$ that are currently technologically feasible are of order of 1 .

Theorem 1 shows that the sizes of bands and gaps of the "worst" spectrum $\sigma_{2}$ are of order $(\varepsilon \delta)^{-1}$. It is natural then, before trying to analyze the spectrum, to zoom in on it by introducing a rescaled spectral parameter $D=(\varepsilon \delta) \lambda$. Then the spectral problem (1) becomes

$$
-\Delta u=(\varepsilon \delta)^{-1} D \varepsilon(x) u .
$$

Consider now the delta-function $\delta_{\Sigma}$ supported by the graph $\Sigma$, i.e., for any compactly supported smooth function $\phi(x)$

$$
\left\langle\delta_{\Sigma}, \phi\right\rangle=\int_{\Sigma} \phi(x) d x .
$$

Theorem 2: (See details in [15]). Any finite part of the spectrum $\sigma$ (in terms of the parameter $D$ ) of the problem (2) converges to the corresponding part of the spectrum of the problem

$$
-\Delta u=D\left(\delta_{\Sigma}+W\right) u .
$$

The constant $W=\lim (\varepsilon \delta)^{-1}$ plays the role of a coupling constant. When $W=0$ the air and dielectric modes decouple. In the latter case, the problem (3) reduces to the transmission problem

$$
-\Delta u=D \delta_{\Sigma} u \text {. }
$$

The natural domain for consideration of this spectral problem is the graph $\Sigma$ itself rather than the whole plane. In order to understand this we need to introduce the notion of the
Dirichlet-to-Neumann operator $(\mathrm{D}-\mathrm{N})$ on the graph $\Sigma$. Take a function $\phi(x)$ defined along the edges of the graph $\Sigma$. Now take any of the compact faces $\Omega_{j}$ and find the harmonic function $u_{j}$ in $\Omega_{j}$ that coincides with $\phi$ on the boundary

$$
\begin{aligned}
-\Delta u_{j}(x) & =0, \quad x \in \Omega_{j} \\
\left.u_{j}\right|_{\partial \Omega_{j}} & =\phi .
\end{aligned}
$$

Repeating this for each face $\Omega_{j}$, we obtain a collection of harmonic functions $u_{j}$. Due to the construction, the values of these functions match across the graph's edges, while their normal derivatives do not match. Let us now define a function $\psi$ on the graph as the sum of all exterior normal derivatives of all the functions $u_{j}$

$$
\psi=\sum_{j} \frac{\partial u_{j}}{\partial n_{j}}
$$

where $\boldsymbol{n}_{\boldsymbol{j}}$ are the exterior normal vectors to $\partial \Omega_{j}$. We define the D-N on the graph $\Sigma$ as the operator

$$
\Lambda_{\Sigma}: \phi \rightarrow \psi
$$

We will abbreviate this name to D-N. It is not hard to show that $\Lambda_{\Sigma}$ is a self-adjoint operator on $L^{2}(\Sigma)$ (see, for instance, [15], [28]).

Theorem 3: [15] The spectrum of the operator $\Lambda_{\Sigma}$ coincides with the spectrum of the problem (4).

This theorem explains the origin of the "bad" spectrum $\sigma_{2}$ : it asymptotically behaves as the spectrum of the D-N operator $\Lambda_{\Sigma}$ rescaled by the small parameter $(\varepsilon \delta)^{-1}$.

The operator $\Lambda_{\Sigma}$ can be thought of as a "pseudo-differential" operator on the graph $\Sigma$. When the graph is smooth (and in particular has no vertices or loose ends) the operator $\Lambda_{\Sigma}$ in fact is a first order pseudo-differential operator in technical sense. Its principal symbol is $2|\xi|$ (i.e., the symbol of the operator $2 \sqrt{-d^{2} / d s^{2}}$, where $s$ is the arc length). Analogously, in the multidimensional situation the operator $\Lambda_{\Sigma}$ is "almost" $2 \sqrt{-\Delta_{\Sigma}}$, where $-\Delta_{\Sigma}$ is the Laplace-Beltrami operator. This understanding is important for what follows. D-N operators have been intensively studied recently, in particular due to the needs of inverse problems (see [36], [37], and references therein). The only thing different in the photonic situation is that the operator $\Lambda_{\Sigma}$ is a "two-sided" one, i.e., we solve Dirichlet problems on both sides of an edge and then take the jump of normal derivatives from both sides, while in standard considerations the Dirichlet problem is solved only on one side, and then the exterior normal derivative at the boundary is taken.

\section{ANALYSIS OF THE ASYMPTOTIC MODEL}

As we saw in the previous section, study of thin highcontrast dielectric structures leads to the spectral problem for the operator $\Lambda_{\Sigma}$ on a periodic graph $\Sigma$ in the plane. A thorough numerical and analytic study of this operator was done in [28] and [29]. Fig. 4 presents the spectrum computed for the square lattice graph formed by the lines $x=n$ and $y=m$ $(n, m \in Z)$ and also explains our graphing system. In this picture the spectral axis is vertical. The first column represents 


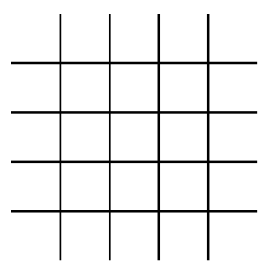

(a)

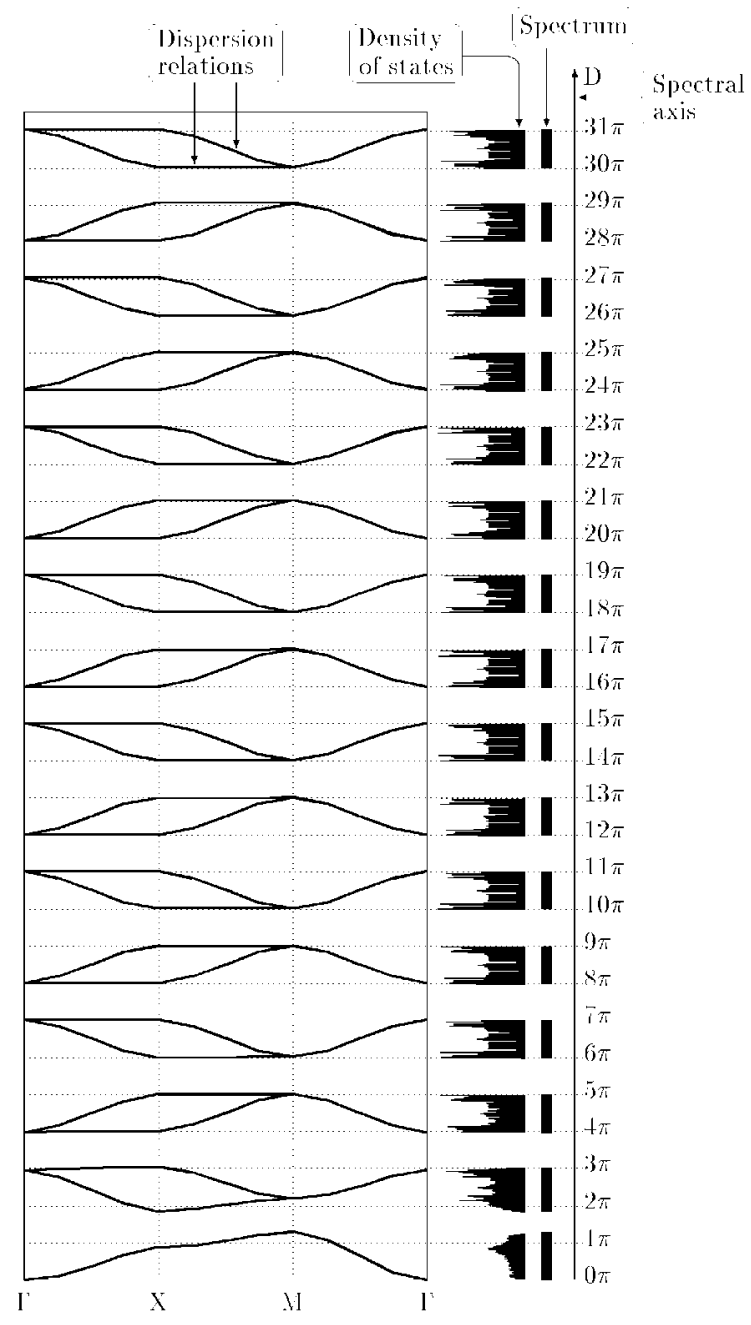

(b)

Fig. 4. (a) Square structure and (b) its spectrum.

the graphs of several branches of the dispersion relations $D_{j}(\boldsymbol{k})$ (we remind the reader that we use here the rescaled spectral parameter $D=(\varepsilon \delta) \lambda=(\varepsilon \delta)(\omega / c)^{2}$ rather than the frequency $\omega)$. The dispersion relation is graphed for the values of the quasi-momentum $\boldsymbol{k}$ on the boundary of the triangle with the vertices $\Gamma(0,0), X(\pi, 0)$, and $M(\pi, \pi)$ (the irreducible Brillouin zone). The second column contains the graph of the density of states over the spectral axis. The third column shows the bandgap structure of the spectrum. One can notice existence of many gaps (in fact, infinitely many of them) and apparent asymptotic periodicity of the dispersion curves with respect to the spectral parameter $D$ (a feature which is hard to catch looking at the graphs in terms of frequencies). These features can be explained and rigorously justified (see [28]).
We will now point out some other interesting features of dispersion curves for different types of dielectric structures. Let us start with disconnected dielectric media. For instance, let us take a circle of a radius less than 0.5 and repeat it periodically with the period group $Z^{2}$. One can view the resulting disconnected graph $\Sigma$ as a model of the structure of thin optically dense dielectric pipes in the air. A similar procedure can be applied to a segment, cross, square, etc., each time yielding a disconnected graph $\Sigma$. The numerical study of all of these and of other disconnected structures produced dispersion relations with band functions that flatten very fast with the growing band number, leading to spectra that consist of very narrow spectral bands and thus are almost discrete for high frequencies. Besides, the spectra appear to be asymptotically periodic. Fig. 5 represents the results of the calculation for the disconnected structure composed of disjoint circles of radii 0.2 .

The reason why the bands flatten like this is that at higher frequencies the modes related to different "pipes" essentially decouple and become significantly localized. Thus the spectrum starts looking numerically like it consists of infinitely degenerate eigenvalues (which in fact are extremely narrow bands).

We present now an analytic result that explains this spectral behavior. It holds in any dimension, not necessarily in 2-D. Let $S$ be a smooth closed hypersurface in $\boldsymbol{R}^{d}$ [a single circle in the Fig. 5(a)] and $\Sigma=\underset{\boldsymbol{n} \in \boldsymbol{Z}^{\boldsymbol{d}}}{\cup}(S+\boldsymbol{n})$ be the disjoint union of the integer shifts of $S$. One can define the D-N operator $\Lambda_{\Sigma}$ on $\Sigma$ as this was done above for $d=2$.

Theorem 4: [28] Let $\left\{D_{n}\right\} \subset \boldsymbol{R}$ be the (discrete) spectrum of the (positive) Laplace-Beltrami operator $-\Delta_{S}$ on the surface $S$. Then there exists a sequence of positive numbers $\rho_{n} \rightarrow 0$ such that the spectrum of the operator $\Lambda_{\Sigma}$ on $\Sigma$ belongs to the union of intervals

$$
\sigma\left(\Lambda_{\Sigma}\right) \subset \bigcup_{n}\left[2 \sqrt{D_{n}}-\rho_{n}, 2 \sqrt{D_{n}}+\rho_{n}\right]
$$

and each of these intervals contains a nonempty portion of $\sigma\left(\Lambda_{\Sigma}\right)$.

In fact, if $S$ is smooth, one can guarantee that $\rho_{n} \leq c_{p} D_{n}^{-p}$ for any $p>0$. The case when $S$ is a circle can be solved explicitly and shows that analyticity of $S$ probably implies exponential decay of $\rho_{n}$.

Theorem 4 explains the "almost discrete" nature of the spectrum and predicts its asymptotic location for disconnected smooth structures. For instance, in the 2-D case, we conclude that the spectrum at higher frequencies must concentrate around values $4 \pi n L^{-1}$, where $L$ is the length of $S$. In particular, for a circle of radius $R$ this leads to $2 n R^{-1}$. These numbers are indicated along the spectral axis in the Fig. 5(b) and one can see perfect agreement with the numerical results. This also provides an explanation of the asymptotic periodicity of the spectrum in 2-D that was observed in numerics.

We would like to mention that numerics shows a very fast convergence of the asymptotics claimed in the last theorem. Therefore, one can make rather accurate predictions about the spectra using this theorem. 


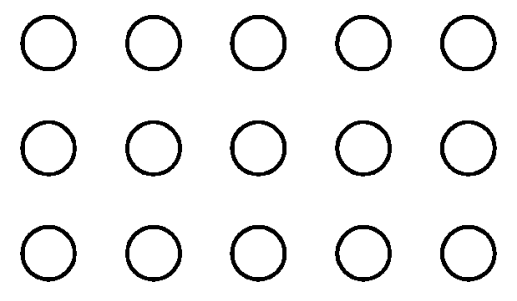

(a)

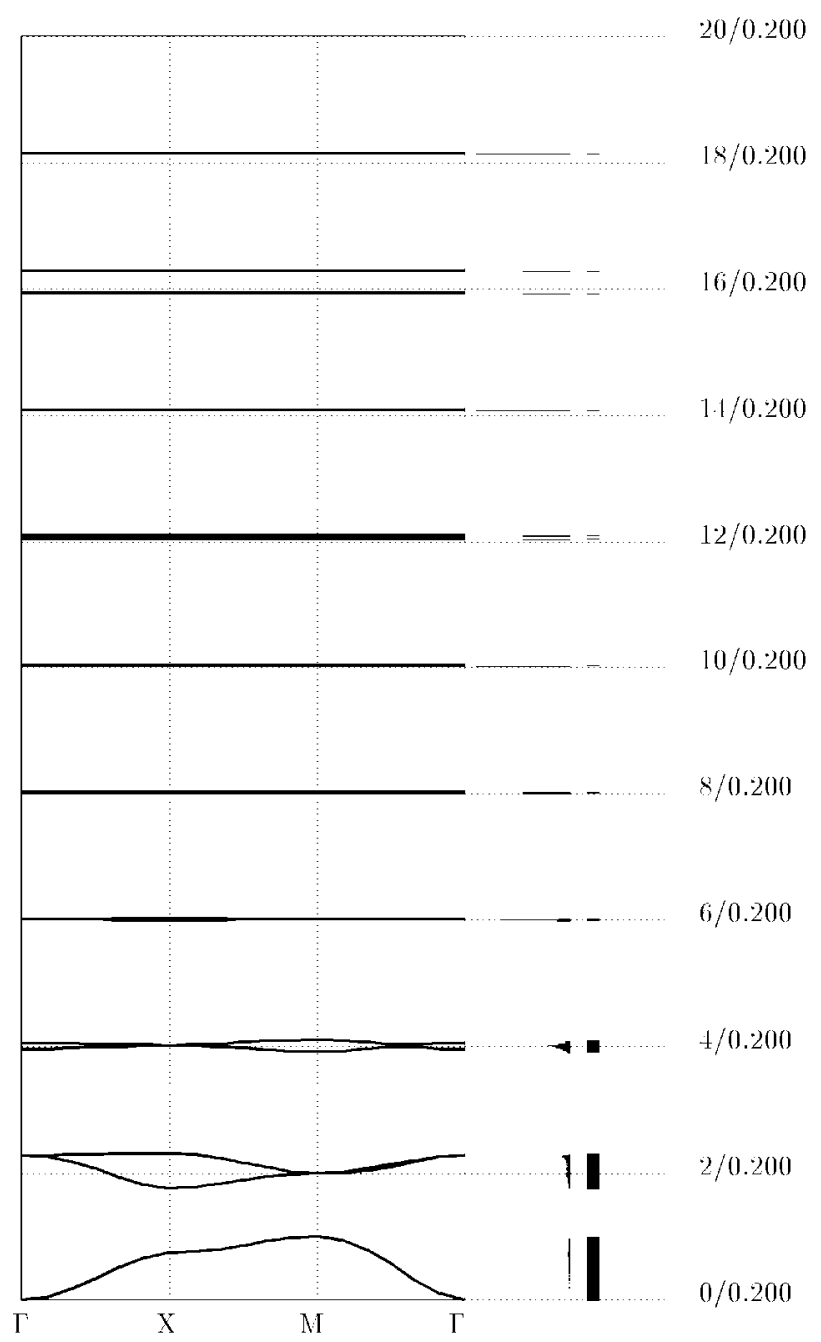

(b)

Fig. 5. (a) Disconnected structure of period 1 consisting of circles of radius 0.2 and (b) its spectrum.

A very restrictive assumption is smoothness of $S$, since graphs that represent thin dielectric structures will normally have vertices and/or corners. One might expect that if instead of circles we use squares of the same length, the asymptotic nature of the spectrum will stay the same. However, numerical tests show that this is not the case. The spectra look systematically shifted from the values calculated according to the formula $4 \pi n L^{-1}$. This effect is due to the singularities at vertices (corners), which require some special boundary conditions. These conditions will be discussed later in Section IV.

Connected (and hence self-supporting) structures are certainly the most interesting ones. The paper [28] contains results

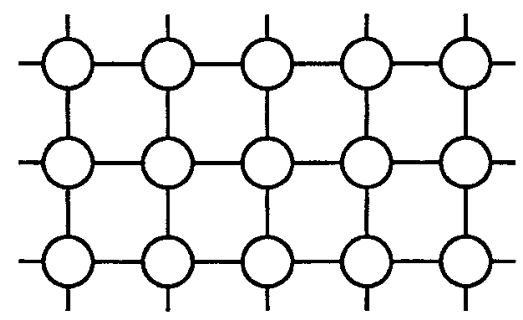

(a)

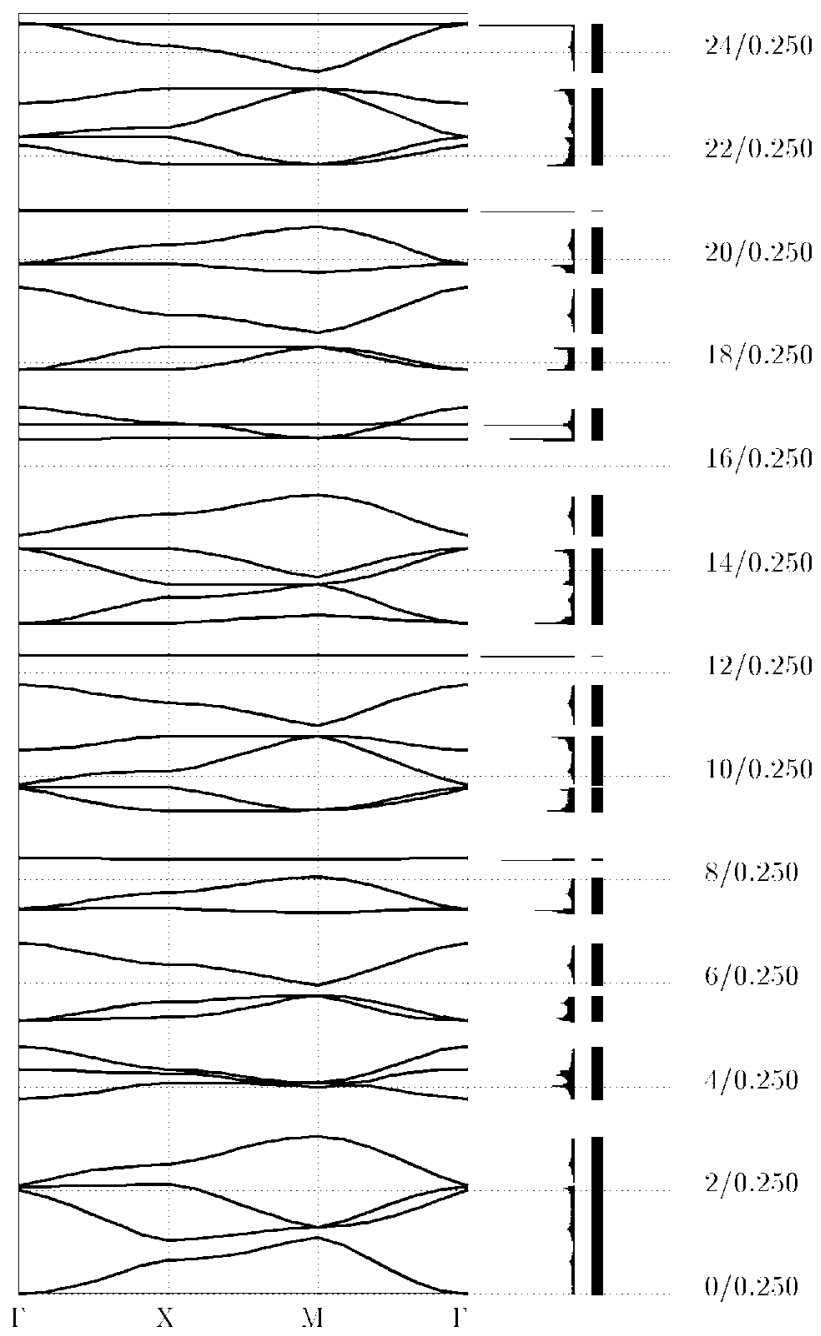

(b)

Fig. 6. (a) Connected circle structure of radii 0.25 and (b) its spectrum.

of computations that show how the spectrum reacts to different geometries. Consider for instance the same disconnected circle structure and add dielectric edges connecting the circles along the symmetry axes of the structure. Fig. 6 represents the computed dispersion relations and spectrum for this model. One can notice that some branches of the dispersion relation become practically flat, and the density of states shows high delta-type peaks at the corresponding locations. This most probably does not indicate presence of actual eigenvalues, but rather of strong resonances.

It is interesting to look at the Floquet-Bloch eigenmodes that correspond to these resonances. Fig. 7 represents a density 

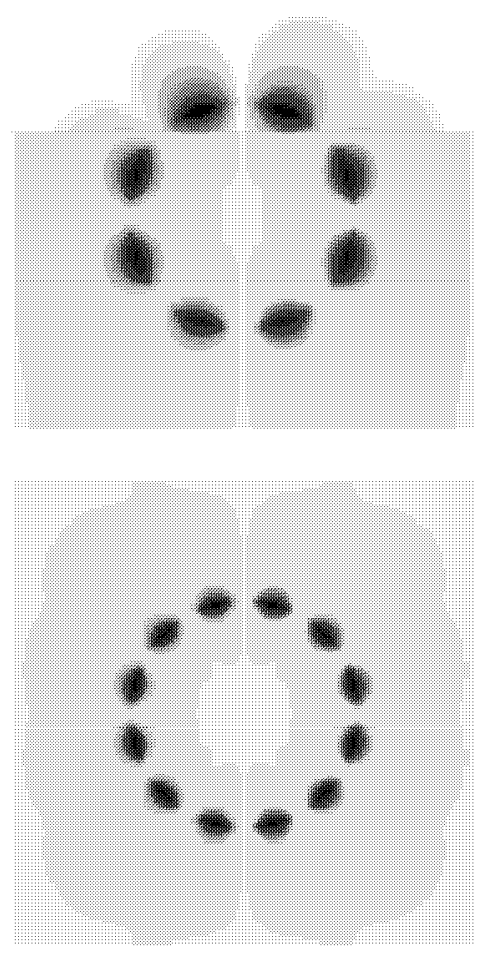

Fig. 7. Density plots of two "localized" eigenmodes for the connected circle structure. The modes apparently do not propagate along the dielectric edges connecting the circles.

plot of a couple of such eigenmodes. What one can see is that the wave is strongly localized at one circle (it is stuck in the loop), in spite of availability of the dielectric edges connecting different circles that allow the wave to propagate. One can also observe that the frequencies at which these resonances occur coincide with a subset of the spectrum computed for the disconnected circle structure. This is not a coincidence. One can show (the corresponding theorem is proven in [28]) that the eigenmodes of the disconnected circle structure that are antisymmetric with respect to both symmetry axes of the structure, lead to resonances in the connected structure. It is interesting to notice that exactly this type of doubly-antisymmetric eigenmodes (and corresponding flat bands) have recently been discovered and used practically in [24]. Similar resonant behavior was also observed in [28] for several other geometries, including for instance the honeycomb one. There is, however, no complete understanding of this effect. For instance, one can show both analytically and numerically that these resonances do not occur in the square geometry. It is not clear yet what differentiates this geometry from those with resonances. The study of these resonances suggests that it is in principle conceivable to "almost localize" electromagnetic waves in a purely periodic PBG material with no impurities, just by using an appropriate geometry. Practical importance of such narrow bands for enhancement of spontaneous emission and lasing was shown in [5] and [24].

The results of [28] show that there are often infinitely many gaps in the spectrum of the D-N operator $\Lambda_{\Sigma}$ on a periodic graph $\Sigma$ in the plane. Is the same true for higher dimensions?

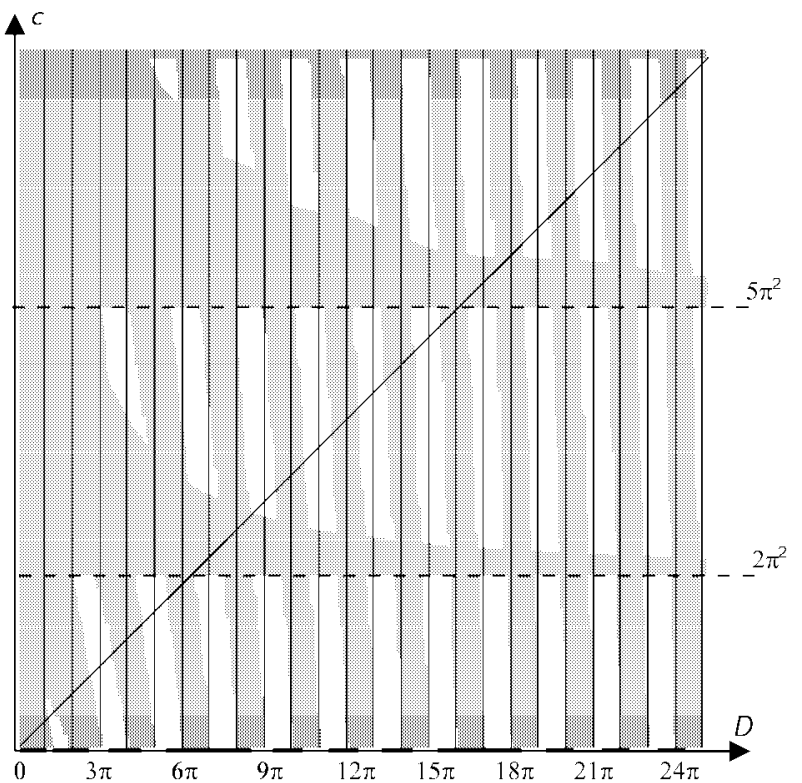

Fig. 8. The spectrum of the problem $-\Delta u-c u=D \delta_{\Sigma} u$ for the square structure.

The following theorem shows that the answer is probably negative.

Theorem 5: [28] Let the space $\boldsymbol{R}^{3}$ be tiled with unit cubes and $\Sigma$ be the union of their surfaces. The spectrum of the corresponding D-N operator $\Lambda_{\Sigma}$ has only a finite number of gaps. Moreover, there are no gaps in the spectrum for the values of the spectral parameter $D \geq 40 \pi$.

We conjecture that the number of gaps in the spectrum of $\Lambda_{\Sigma}$ is finite for any periodic hypersurface structure $\Sigma \subset R^{d}$, $d \geq 3$.

Let us address now the asymptotic problem with $W \neq 0$

$$
-\Delta u=D\left(\delta_{\Sigma}+W\right) u
$$

In this case, there is the dielectric-air coupling, and the problem cannot be conveniently reduced to the graph $\Sigma$. One of the ways one can handle this is to consider the auxiliary problem with two spectral parameters $c$ and $D$

$$
-\Delta u-c u=D \delta_{\Sigma} u
$$

and then to intersect its spectrum in the $(c, D)$-plane with the line $c=W D$. The computation of the spectrum was done by fixing $c$, using the Green's function to rewrite the problem on $\Sigma$, and finally finding numerically the spectrum with respect to $D$. Doing so for many values of $c$, one can recover the 2-D spectrum of the problem. Fig. 8 represents the results of such calculation for the square structure (i.e., formed by the lines $x=n$ and $y=m, m, n \in Z$ ). Similar studies have been done for other geometries as well. The $D$-axis is horizontal and the $c$-axis is vertical. The shaded areas show the 2-D spectrum and the inclined line is $c=D$. One can see that the $(c, D)$ spectrum shows two distinct patterns. First of all, almost vertical strips originate at $c=0$ from the bands of the spectrum of the $\mathrm{D}-\mathrm{N}$ operator. Another set of narrowing strips goes in horizontal direction. The horizontal lower edges of these 
strips indicate that at the corresponding values of $c$ the $D$ spectrum of the problem (5) degenerates and covers the whole line. The two different patterns intersect the line $c=D$ over two different subspectra, which correspond to the subspectra $\sigma_{1}$ (horizontal strips) and $\sigma_{2}$ (vertical strips), respectively. These are exactly the two subspectra that were discussed in the previous section. The next result (applicable to a general "fattened graph" geometry) provides a simple criterion of when the spectral degeneration observed at the straight edges of the horizontal strips can occur. This can provide guidance for creating geometry in a way that eliminates or lifts the horizontal pattern higher.

Theorem 6: ([29]) The degeneration observed on the picture occurs at a level $c$ if and only if

i) $c$ is in the spectrum of the Floquet Laplacian $-\Delta_{k}=$ $(-i \nabla+k)^{2}$ on the torus for some real value of the quasimomentum $k$;

ii) the graph $\Sigma$ belongs to the nodal set of an eigenfunction $\phi$ of $-\Delta_{k}$ corresponding to the eigenvalue $c$.

\section{ORDINARY DIFFERENTIAL EQUATIONS ON GRAPHS AND PBG MATERIALS}

Problems in thin domains (fattened graphs or surfaces) have been considered lately in several areas of mesoscopic physics and in chemistry, mostly due to progress in nanotechnology and microelectronics. These are in particular studies of circuits of thin semiconductor or superconducting strips, models of organic molecules, and others (see, for instance, [2], [3], [8]-[10], [18]-[20], [31]-[34]). In all these cases a natural asymptotic consideration was applied, which lead to differential problems on graphs (sometimes called "quantum mechanics on graphs"). The eigenvalue problems that arise in these studies usually look as follows: along each edge of the graph one has the problem

$$
-\frac{d^{2} u}{d s^{2}}=\lambda^{2} u
$$

with "appropriate" boundary conditions at each vertex.

As we have indicated above, the operator $\Lambda_{\Sigma}$ that arises when one studies thin high-contrast dielectrics, is a pseudodifferential operator of order one, while in mesoscopic physics one deals with differential operators of the second order. However, there sometimes exists an amazing possibility to reduce the consideration to a much simpler ordinary differential problem on the graph. The first indication of this is the case of smooth disconnected structures. Theorem 4 essentially reduces consideration of the operator $\Lambda_{\Sigma}$ to the simple differential operator $-d^{2} / d s^{2}$ (roughly speaking, $\Lambda_{\Sigma} \approx 2 \sqrt{-d^{2} / d s^{2}}$ ) and leads to the approximate formula $D \approx 4 n \pi / L$. However, the most interesting case of nonsmooth graphs $\Sigma$ is not so simple. The question is whether a statement analogous to the Theorem 4 holds in the nonsmooth case. An attempt to use the formula $D \approx 4 n \pi / L$ for nonsmooth disconnected structures (for instance, the structure of thin dielectric pipes of square cross section) leads to results that do not agree well with numerics. One can hope, however, that appropriate boundary conditions at vertices and/or corners could fix the situation. Namely, since the D-N operator is "almost" twice the square root of the second derivative with respect to the arc length $-d^{2} / d s^{2}$, it is clear that it is reasonable to consider the eigenvalue problem

$$
-\frac{d^{2} u}{d s^{2}}=\left(\frac{D}{2}\right)^{2} u
$$

along each edge (or maybe

$$
(-1)^{m} \frac{d^{2 m} u}{d s^{2 m}}=\left(\frac{D}{2}\right)^{2 m} u
$$

for some integer $m$ ). The question arises, however, what boundary conditions at the vertices and corners one should use. Although the general answer is not known, some special geometries can be treated. Analysis developed in [29], although not being completely rigorous, provides an interesting and reliable heuristic technique. In order to understand the boundary behavior of an eigenmode at a vertex or corner, one zooms in on the vertex by applying the Mellin transform in the radial directions. Then the problem can be reduced to studying an interesting algebra of poles of analytic continuation of the resulting function. The spectral problem for the $\mathrm{D}-\mathrm{N}$ operator provides a functional equation that can be used to study these singularities. We will just present one of the results that can be obtained this way. The complete discussion will be provided in [29]. If one has a symmetric junction of three edges at a vertex and $u_{j}$ is the restriction of the function to the $j$ th edge, then our analysis leads to the following conditions at the vertex:

$$
\begin{aligned}
& u_{1}(0)=u_{2}(0)=u_{3}(0) \\
& \sum_{j=1,2,3} \frac{d u_{j}}{d s}(0)=-\left(\frac{3-D}{2} \cot \frac{\pi}{3}\right) u(0) .
\end{aligned}
$$

An interesting feature here (besides a funny trigonometric factor) is that the spectral parameter $D$ also enters the boundary conditions. Tests on the disconnected union of threeedge stars, honeycomb structures, and some other geometries lead to an amazing agreement between the differential and pseudo-differential results. The problem (6) leads to simple algebraic equations and hence in many cases can be analyzed analytically. For instance, the dispersion relations for (6) in the case of the honeycomb structure with the edge size $L$ can be found explicitly. Namely, one can derive existence of a series of eigenvalues $D=2 n \pi / L$ and of a series of nonflat band functions given by

$$
\begin{aligned}
D_{n}(\boldsymbol{k})= & \frac{2}{L}\left(\pi n+\frac{\pi}{3}\right. \\
& \left. \pm \arcsin \sqrt{\frac{1}{4}+\frac{1}{6} \cos k_{2} \pm \frac{1}{3} \cos \frac{k_{1}}{2} \cos \frac{k_{2}}{2}}\right) .
\end{aligned}
$$

Existence of eigenvalues for a spectral problem in a purely periodic medium is a highly unusual thing. One can also find occurrence of eigenvalues in similar situations of periodic meshes of quantum wires (a circumstance overlooked in previous studies of such systems). Certainly, for actual photonic situations (i.e., before going to the asymptotic limit) these eigenvalues correspond to very flat bands and hence 


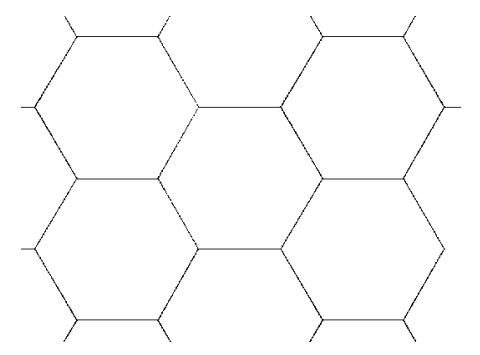

(a)

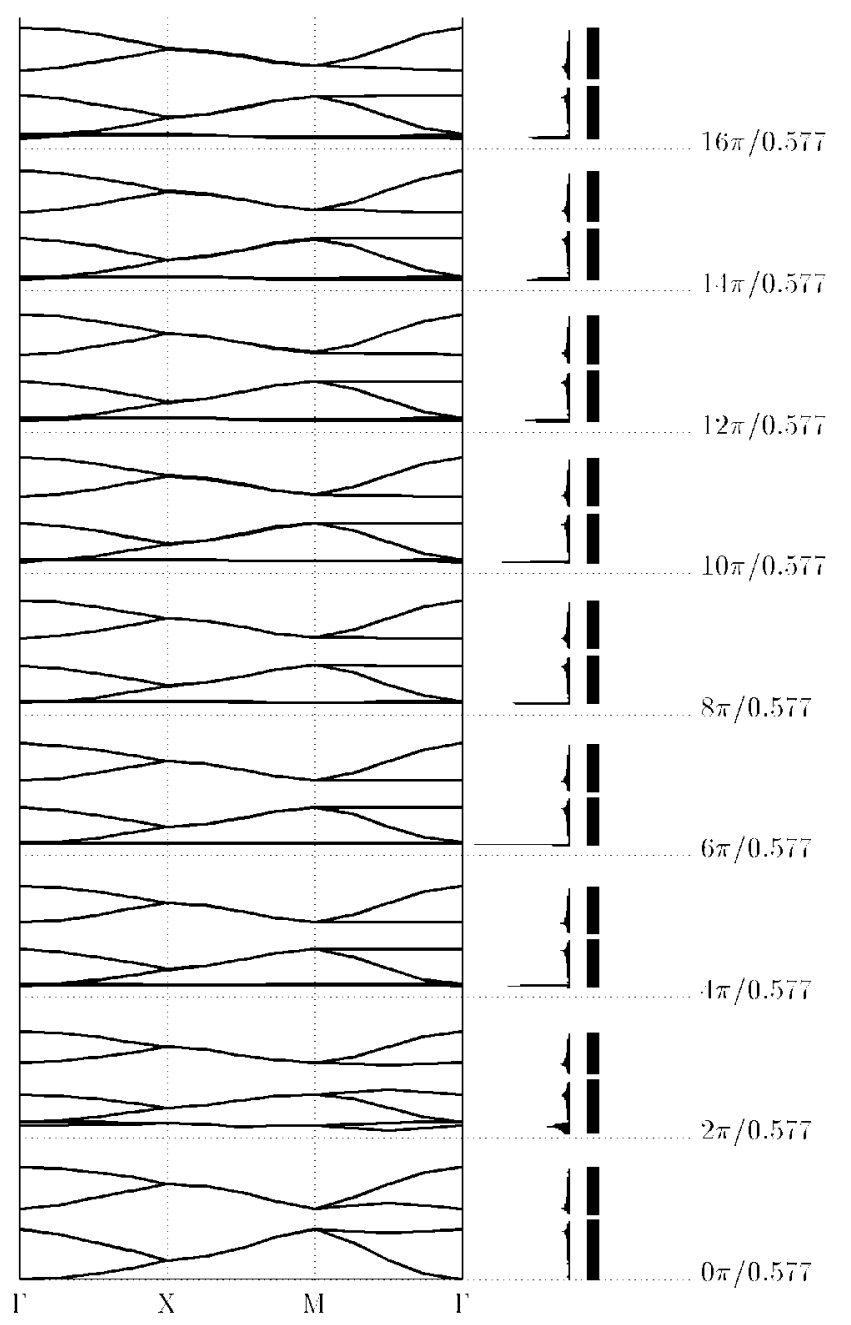

(b)

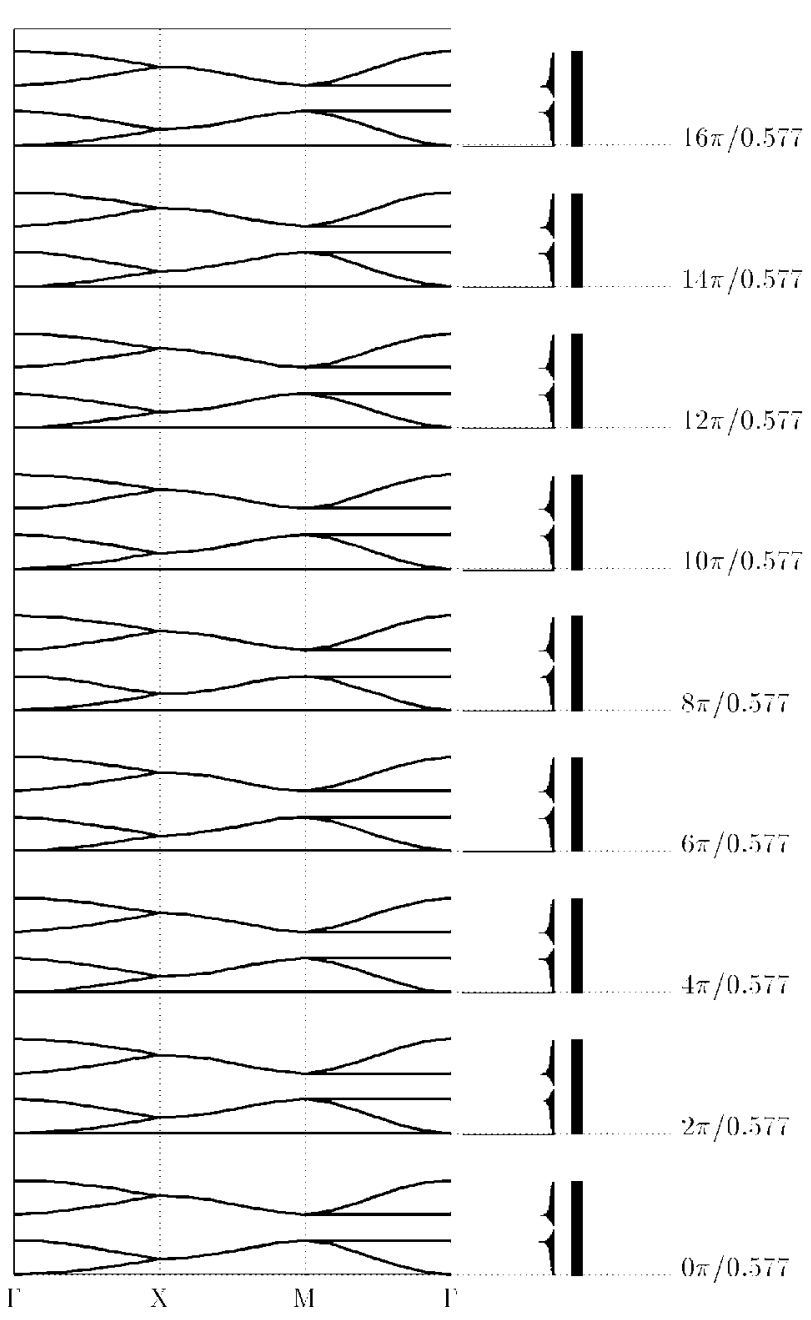

(c)

Fig. 9. (a) Honeycomb structure, (b) spectrum of the corresponding D-N operator $\Lambda$, and (c) spectrum of the differential model.

to resonances. Fig. 9 presents the results of computing the spectrum using the differential model (6) and the pseudodifferential operator $\Lambda_{\Sigma}$ for the honeycomb lattice in the plane. One can see a striking agreement between them. The pictures differ a little bit for the lowest band functions, but otherwise are practically identical. No rigorous justification of this effect is known.

Analytic formulas similar to (7) can be derived for some other structures, for instance for the octagonal structure discussed in the next section.

\section{How GoOD Is THE ASYMPTOTICS?}

The asymptotic results that we discussed above are derived under the assumptions that $\delta \rightarrow 0$ and $\varepsilon \delta \rightarrow \infty$ (or a little bit less restrictive assumption $\varepsilon \delta \rightarrow W^{-1}>0$ ). On the other hand, the currently practically feasible values of $\delta$ and $\varepsilon$ are such that $\varepsilon \delta \simeq 1$ (although in the acoustic case one can reach much higher contrasts). The question arises whether the asymptotic study can give any guidance for these values of the parameters. A rather unexpected observation is that the 


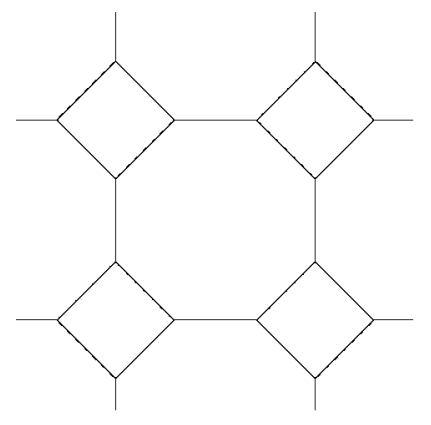

Fig. 10. Octagonal structure.

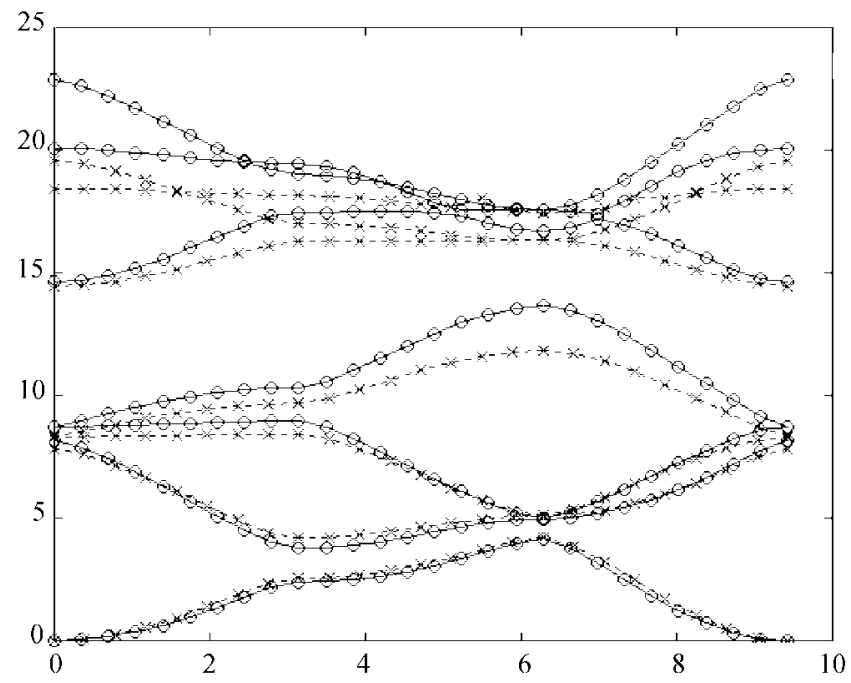

Fig. 11. Asymptotic results (marked with " $\times$ ") and the actual computation (marked with "o") for the octagonal structure.

asymptotic results (even when $\varepsilon \delta$ is assumed to tend to infinity) can give a good qualitative, and even some quantitative idea about the behavior of a several lower spectral bands. We plan to present a more detailed discussion of this matter elsewhere, and now we just provide some examples. Consider for instance the periodic geometry of octagons intertwined with squares with all edges of equal length (Fig. 10). We remind the reader that the edges of the graph $\Sigma$ represent thin dielectric walls in the air. Fig. 11 represents the comparison of the computations of the dispersion relations using the asymptotic model (the D-N operator $\Lambda_{\Sigma}$ ) and computations performed with the finite element method developed in [4] for the similar structure with the dielectric walls of width 0.05 (relative to the period of the structures) and of the dielectric constant $\varepsilon=30$. The asymptotic results are marked with " $x$ " and the actual ones with "o." One observes a perfect match for the first three bands, some deviation for the fourth one, and the next three bands more significantly distorted (albeit preserving their qualitative geometric features). Taking into account that we used the asymptotic model with $\varepsilon \delta \rightarrow \infty$ for the actual situation where $\varepsilon \delta=1.5$, the agreement is very surprising. It is clear that the asymptotic models can provide a good guidance for understanding the spectral behavior. One should also remember that in many cases the asymptotic model yields simple analytic formulas for dispersion relations. The results

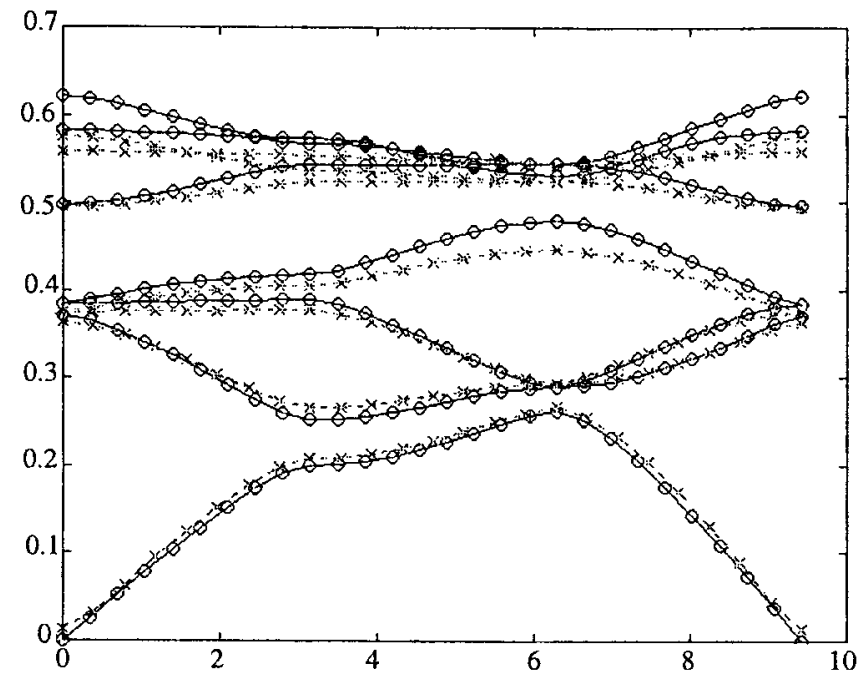

Fig. 12. The same results as in Fig. 11 redrawn for the frequency $\omega a / 2 \pi c$ instead of $\lambda$.

become even more impressive if one graphs the frequency (in standard dimensionless units $\omega a / 2 \pi c$ ), as it is shown in Fig. 12. The reader should take into account that Fig. 12 through Fig. 15 present results in terms of the frequency $\omega$ rather than $\lambda$.

Comparison of actual and asymptotic calculations show that asymptotic results have value even for the case of $\delta=0.1$ and $\varepsilon=12$. Consider the hexagonal structure shown in Fig. 3(a). The length of a single edge was chosen to be one-third. Consider now the dielectric structure in Fig. 3(b). We chose the values $\delta=0.1$ and $\varepsilon=12$. The reader should notice that this situation is very far from the asymptotic assumption of a very thin dielectric with high-contrast, since the filling fraction of the dielectric is as high as 0.32 , and $\varepsilon \delta=1.2$. The explicit formulas (7) together with the corresponding formulas for the flat bands were applied to the graph of Fig. 3(a) and simultaneously a direct numerical calculation was performed for the PBG material of Fig. 3(b). The results of both computations are presented (in arbitrary frequency units) in Fig. 13. One notices that although the numerical values are not too close (albeit not extremely far off), the overall pattern of the spectrum is captured by the formulas (7) very well for the first 9-10 bands. Numerical experiments with increasing the contrast to 30 and 100 while keeping the filling fraction constant produce results that show about the same level of agreement. However, reducing simultaneously the value of $\delta$ one improves the agreement. Fig. 14 shows the results of similar calculations for $\delta=0.05$ and $\varepsilon=30$. Here, the filling fraction of the dielectric is only about 0.17 . One can see that the overall pattern is represented well. This can also be checked as follows. One can rescale the values of $\lambda=(\omega / c)^{2}$ by a constant factor (about 1.2) in order to make the flat bands $\{\# \#\} 5$ and 6 meet in both computations. Then one sees perfect agreement of all other bands as well (Fig. 15).

\section{AN ASYMPtotic Model FOR THE $H$-FieldS IN 2-D}

So far, our discussion has been devoted to the case of $E$-modes only. Consider now the behavior of the spectrum 


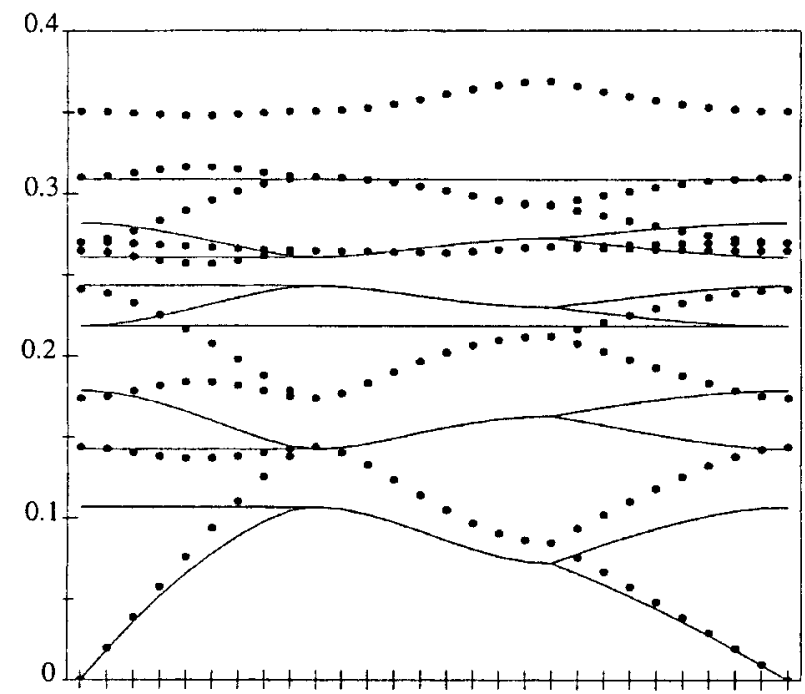

Fig. 13. Dispersion curves $\omega(k)$ obtained by using the explicit formulas [7] (solid lines) and direct computations (dotted lines) for the hexagonal structure of Fig. 3(b) with $L=1 / 3, \delta=0.1$, and $\varepsilon=12$.

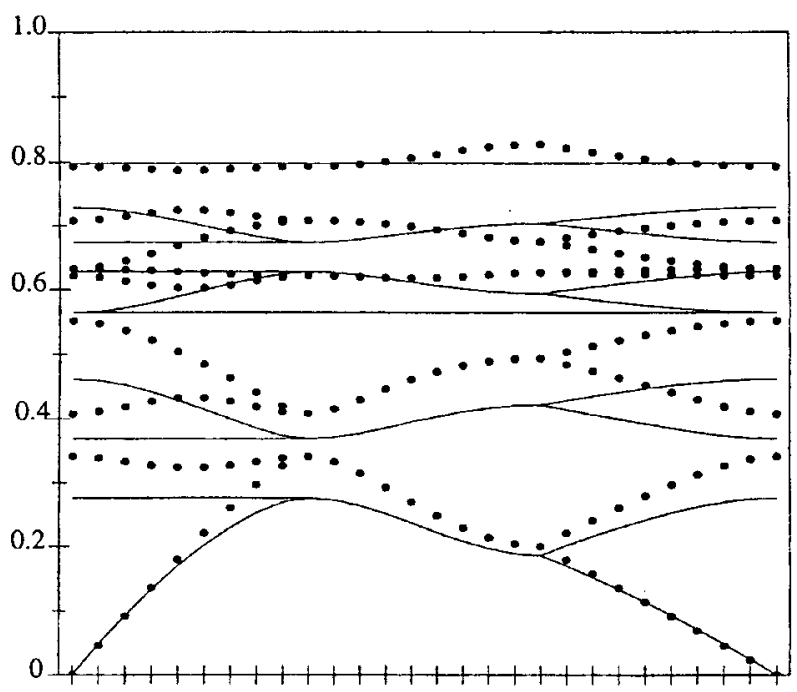

Fig. 14. Results similar to the ones in Fig. 13 for $\delta=0.05$ and $\varepsilon=30$.

of $H$-polarized fields in thin high-contrast 2-D materials. For these fields the Maxwell system reduces to the spectral problem

$$
-\nabla \cdot \frac{1}{\varepsilon(x)} \nabla u=\lambda u
$$

where $\lambda=(\omega / c)^{2}$. Let $\sigma$ be the spectrum of the problem (8). The paper [12] describes the asymptotic limit of this spectrum when $\varepsilon \delta \rightarrow \infty$ and $\varepsilon \delta^{2} \rightarrow 0$ for the square geometry shown in Fig. 1.

Theorem 7: [12] Let $N$ be an arbitrary positive number and

$$
S_{1}=\left\{\pi^{2}\left(n_{1}^{2}+n_{2}^{2}\right) \mid n=\left(\boldsymbol{n}_{1}, \boldsymbol{n}_{\mathbf{2}}\right) \in \boldsymbol{Z}^{2}=\right\}
$$

be the spectrum of the Neumann Laplacian on the unit square (which is the Wigner-Seitz cell of the considered geometry). Then the Hausdorff distance between $\sigma \cap[0, N]$ and $S_{1} \cap[0, N]$ tends to zero when $\varepsilon \delta \rightarrow \infty$ and $\varepsilon \delta^{2} \rightarrow 0$.

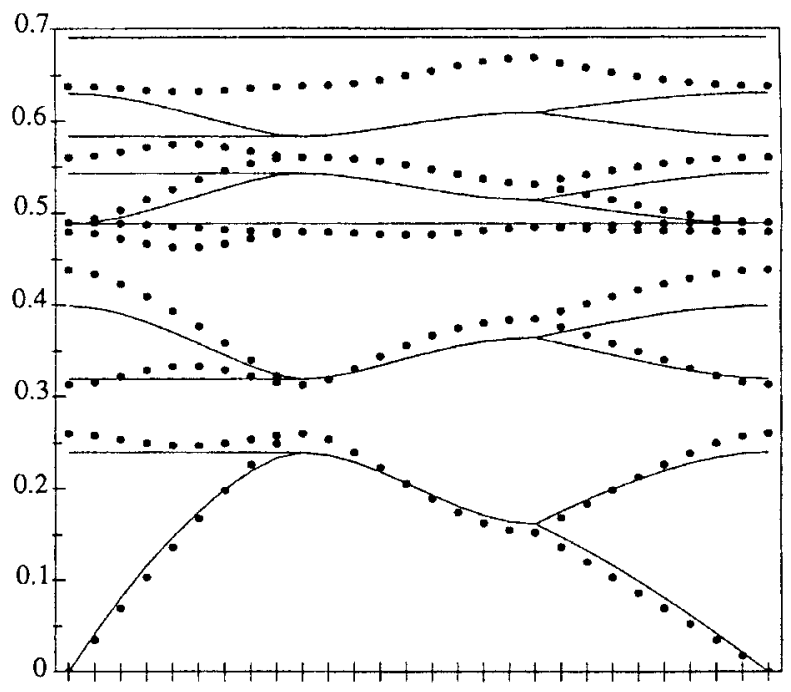

Fig. 15. Results shown in Fig. 14 after rescaling the spectral parameter $\lambda=(\omega / c)^{2}$ by a factor of 1.2 .

Moreover,

$$
d\left(S_{1} \cap[0, N], \sigma \cap[0, N]\right) \leq C_{N} \max \left\{(\varepsilon \delta)^{-1}, \varepsilon \delta^{2}\right\}
$$

where $d$ denotes the Hausdorff distance.

This theorem says that the spectrum of the $H$-fields for small values of $(\varepsilon \delta)^{-1}$ and $\varepsilon \delta^{2}$ concentrates in a small vicinity of the discrete set $S_{1}$, and hence, large gaps at exactly known locations open up. A more precise description of this result can be found in [12]. An additional observation made in [12] was that the eigenmodes have most of their energy concentrated in the air. We would also like to mention that the same result holds for the problem (8) for the cubic geometry in three-dimensional (3-D) [12], where one can think of (8) as describing acoustic rather than EM waves.

The paper [16] considers the asymptotics of the $H$-fields in 2-D for arbitrary "fattened graph" geometry and under the milder condition $\delta \rightarrow 0, \varepsilon \delta \rightarrow W^{-1}>0$. It is shown, in particular, that the spectrum converges to the spectrum of the following problem:

$$
\begin{aligned}
-\Delta u & =\lambda u, \quad x \in R^{2}-\Sigma \\
{\left[\frac{\partial u}{\partial n}\right] } & =0, \quad x \in \Sigma \\
\frac{\partial u}{\partial n} & =W[u], \quad x \in \Sigma
\end{aligned}
$$

where $[(\partial u / \partial n)]$ and $[u]$ stand for the jumps of $\partial u / \partial n$ and of $u$, respectively, across $\Sigma$ and $\partial / \partial n$ is the normal derivative at smooth points of $\Sigma$. Note that the surface term with the coefficient $W$ couples the fields in adjacent domains $\Omega_{j}$. When $W$ tends to zero, we arrive to the direct sum of the Neumann Laplacians in the domains $\Omega_{j}$, which agrees with the result of the Theorem 7 derived in [12] for the square structure and $W=0$. Additional analytic and numerical study of this asymptotic problem is still required. Some analogs of this asymptotic result for EM waves in 3-D will be presented in [17]. 
One should also mention the paper [22] devoted to the asymptotic study of a high-contrast case without the assumption of thinness of the dielectric.

\section{CONCLUSIONS}

1) Rigorous asymptotic models for spectra of EM waves in 2-D thin high-contrast dielectrics are available.

2) These models allow discovery and study of new interesting phenomena, including flat branches of dispersion curves that can be used for spontaneous emission enhancement, gaps in the long wave regions, and asymptotic periodicity of the spectrum.

3) The asymptotic results provide unexpectedly good qualitative (and sometimes quantitative) description of the spectral behavior for materials of finite width and contrast.

4) In some cases very simple ordinary differential models can be derived that provide a good approximation of spectra. In these situations one can often obtain approximate analytic formulas for the dispersion relations.

5) The developed asymptotic results can be used in different ways. For instance, they provide some simple tools of fast estimates of spectral behavior and make it possible to analyze some problems analytically rather than purely numerically. They could also provide good preconditioners for existing iterative numerical methods like for instance the ones developed in [4] and [7].

\section{ACKNOWLEDGMENT}

The authors express their gratitude to Prof. J. W. Haus and Prof. R. Hempel, Dr. D. Sievenpiper, and graduate students M. Boroditsky, R. Vrijen, and H. Zeng for information about their work and discussion. P. Kuchment is indebted to Prof. A. Figotin for numerous discussions and collaboration. The authors thank the NSF, ARO, and the state of Kansas for their support.

\section{REFERENCES}

[1] N. W. Ashcroft and N. D. Mermin, Solid State Physics. New York: Holt, Rinehart and Winston, 1976.

[2] Y. Avishai and J. M. Luck, "Quantum percolation and ballistic conductance on a lattice of wires," Phys. Rev., vol. B45, pp. 1074-1095, 1992.

[3] J. E. Avron, A. Raveh, and B. Zur, "Adiabatic transport in multiply connected systems," Rev. Mod. Phys., vol. 60, pp. 873-915, 1988.

[4] W. Axmann and P. Kuchment, "An efficient finite element method for computing spectra of photonic and acoustic band-gap materials I. Scalar case," J. Comput. Phys., vol. 150, pp. 468-481, 1999

[5] M. Boroditsky, R. Vrijen, T. Krauss, R. Coccioli, R. Bhat, and E. Yablonovich, "Control of spontaneous emission in photonic crystals," preprint 1998

[6] C. M. Bowden, J. P. Dowling, and H. O. Everitt, Eds., "Development and applications of materials exhibiting photonic band gaps," J. Opt. Soc. Amer. B, vol. 10, pp. 280-413, 1993.

[7] D. C. Dobson, "An efficient method for band structure calculations in 2-D photonic crystals," J. Comp. Phys., vol. 149, pp. 363-376, 1999.

[8] P. Exner and P. Seba, "Electrons in semiconductor microstructures: A challenge to operator theorists," in Proc. Workshop Schrödinger Operators, Standard and Nonstandard (Dubna 1988), P. Exner and P. Seba, Eds. Singapore: World Scientific, 1989, pp. 79-100.

[9] __ "Free quantum motion on a branching graph," Rep. Mod. Phys., vol. 28 , pp. 7-26, 1989.
[10] P. Exner, "Contact interactions on graph superlattices," J. Phys., vol. A29, pp. 87-102, 1996.

[11] A. Figotin and A. Klein, "Localization of light in lossless inhomogeneous dielectrics," J. Opt. Soc. Amer. A, vol. 15, no. 5, pp. 1423-1435, 1998.

[12] A. Figotin and P. Kuchment, "Band-gap structure of spectra of periodic and acoustic media. I. Scalar model," SIAM J. Appl. Math., vol. 56, no. 1, pp. 68-88, 1996.

[13], "2-D photonic crystals with cubic structure: Asymptotic analysis," in Wave Propagation in Complex Media, G. Papanicolaou, Ed., IMA volumes in Math. and Appl., vol. 96, p. 23-30, 1997.

[14] _ ,Band-gap structure of spectra of periodic and acoustic media. II 2-D photonic crystals," SIAM J. Appl. Math., vol. 56, pp. 1561-1620, 1996.

[15] _ "Spectral properties of classical waves in high contrast periodic media," SIAM J. Appl. Math., vol. 58, no. 2, pp. 683-702, 1998

[16] _ "Asymptotic models of high contrast periodic photonic and acoustic media. I," in preparation.

[17] _ "Asymptotic models of high contrast periodic photonic and acoustic media. II," in preparation.

[18] M. Freidlin and A. Wentzell, "Diffusion processes on graphs and the averaging principle," Ann. Probability, vol. 21, no. 4, pp. 2215-2245, 1993.

[19] N. Gerasimenko and B. Pavlov, "Scattering problems on noncompact graphs," Theor. Math. Phys., vol. 75, pp. 230-240, 1988, in Russian.

[20] J. Gratus, C. J. Lambert, S. J. Robinson, and R. W. Tucker, "Quantum mechanics on graphs," J. Phys., vol. A27, pp. 6881-6892, 1994.

[21] J. W. Haus, M. Scalora, M. J. Bloemer, J. P. Dowling, and C. M. Bowden, "Photonic crystals review," to appear in Mathematical Modeling in Optical Science. Philadelphia, PA: SIAM, 1999.

[22] R. Hempel and K. Lienau, "Spectral properties of periodic media in the large coupling limit," Commun. PDE, to be published.

[23] P. M. Hui and N. F. Johnson, "Photonic band-gap materials," in Solid State Phys., H. Ehrenreich and F. Spaepen, Eds. New York: Academic, vol. 49, pp. 151-203, 1995.

[24] K. Inoue, M. Sasada, J. Kawamata, K. Sakoda, and J. W. Haus, "A two-dimensional photonic crystal laser," Japan J. Appl. Phys. 2, vol. 38, no. 199, pp. L157-L159.

[25] J. D. Jackson, Classical Electrodynamics. New York: Wiley, 1962.

[26] J. D. Joannopoulos, R. D. Meade, and J. N. Winn, Photonic Crystals. Molding the Flow of Light. Princeton, NJ: Princeton University Press, 1995.

[27] P. Kuchment, "The mathematics of photonic crystals," to appear in Mathematical Modeling in Optical Science. Philadelphia, PA: SIAM, 1999.

[28] P. Kuchment and L. Kunyansky, "Spectral properties of high contrast band-gap materials and operators on graphs," Exp. Math., vol. 8, no. 1, pp. 1-28, 1999

[29] P. Kuchment, L. Kunyansky, and H. Zeng, "Spectral properties of high contrast band-gap materials and operators on graphs," Part II, in preparation.

[30] J. Rarity and C. Weisbuch, Eds., Microcavities and Photonic Bandgaps: Physics and Applications, in Proceedings of the NATO Advanced Study Institute: Quantum Optics in Wavelength-Scale Structures, Cargese, Corsica, Aug. 26-Sept. 2, 1995, NATO ASI Series, Kluwer, The Netherlands, 1996.

[31] J. Rubinstein and M. Schatzman, "Asymptotics for thin superconducting rings," J. Math. Pures Appl., vol. (9) 77, no. 8, pp. 801-820, 1998

[32] _ "On multiply connected mesoscopic superconducting structures," in Séminaire de Théorie Spectrale et Géométrie, no. 15, Année 1996-1997, 207-220, Sémin. Théor. Spectr. Géom., 15, Univ. Grenoble I, Saint-Martin-d'Hères, 1998.

[33] K. Ruedenberg and C. W. Scherr, "Free-electron network model for conjugated systems. I. Theory," J. Chem. Phys., vol. 21, no. 9, pp. 1565-1581, 1953.

[34] M. Schatzman, "On the eigenvalues of the Laplace operator on a thin set with Neumann boundary conditions," Appl. Anal., vol. 61, pp. 293-306, 1996

[35] C. M. Soukoulis, Ed., Photonic Bands and Localization. New York: Plenum, 1993.

[36] J. Sylvester and G. Uhlmann, "Inverse boundary value problems at the boundary-continuous dependence," Commun. Pure Appl. Math., vol. XLI, pp. 197-219, 1988

[37] G. Uhlmann, "Inverse boundary value problems and applications," Asterisque, vol. 207, pp. 153-211, 1992.

[38] P. R. Villaneuve and M. Piché, "Photonic band gaps in periodic dielectric structures," Progr. Quantum Electron., vol. 18, pp. 153-200, 1994.

[39] E. Yablonovitch, "Inhibited spontaneous emission in solid-state physics and electronics," Phys. Rev. Lett., vol. 58, p. 2059, 1987. 
Wally Axmann received the B.S. degree in physics from Kansas State University, Manhattan, KS, in 1991 and the M.S. degree in mathematics from Wichita State University, Wichita, KS, in 1998. During the academic year 1998-1999, he was a Ph.D. student in the applied mathematics program at Wichita State University, Wichita, KS. He is currently pursuing the Ph.D. degree in physics at Kansas State University, Manhattan.

Peter Kuchment received the $\mathrm{Ph} . \mathrm{D}$. degree in mathematics from Kharkov State University, Kharkov, Ukraine, in 1973 and the D.Sc. degree in mathematics from the Mathematics Institute of Academy of Sciences, Kiev, Ukraine, in 1983.

$\mathrm{He}$ is a Professor at the Mathematics and Statistics Department, Wichita State University, Wichita, KS. His current research interests include mathematical physics, photonic crystals, computerized tomography, integral geometry, and K-12 education.
Leonid Kunyansky received the Ph.D. degree in engineering from Kharkov Polytechnic University, Kharkov, Ukraine, in 1993 and the Ph.D. degree in applied mathematics from Wichita State University, Wichita, KS, in 1999.

$\mathrm{He}$ is currently holds a Postdoctoral position at the Applied Mathematics Department, California Institute of Technology, Pasadena. His research experiences and interests include mathematical physics, numerical analysis, computerized tomography, and scientific computing. 